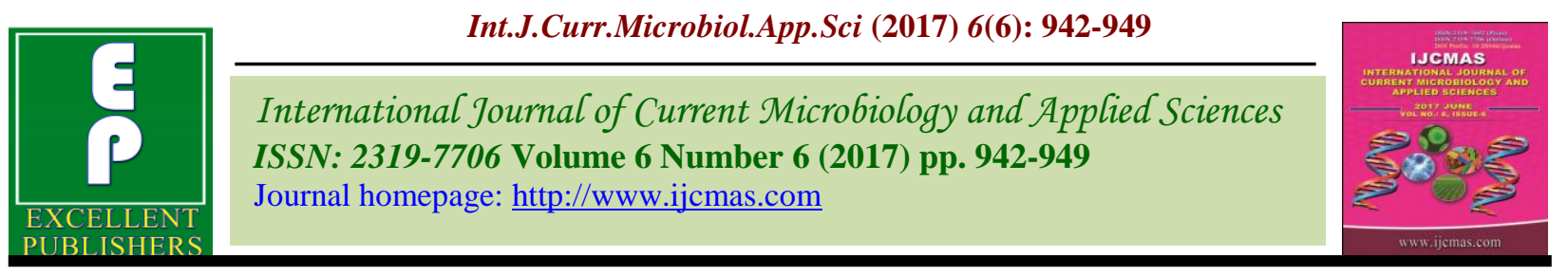

Original Research Article

https://doi.org/10.20546/ijcmas.2017.606.110

\title{
Combining Ability Studies on Cucumber and Snapmelon Hybrids
}

\author{
Sheetal Tak*, R.A. Kaushik, K.D. Ameta, R.B. Dubey, R.S. Rathore and Anamika Nath \\ Rajasthan College of Agriculture, MPUAT, Udaipur, Rajasthan -313001, India \\ *Corresponding author
}

\begin{abstract}
A B S T R A C T
\end{abstract}
A field study with 12 × 3 Line $\times$ Tester analysis in cucumber and

Keywords

Cucumber,

GCA, SCA,

F1 hybrids,

Line $\mathrm{x}$ testers.

Article Info

Accepted:

17 May 2017

Available Online:

10 June 2017 snapmelon revealed highly significant estimates for general combining ability and specific combining ability for all the traits thereby indicating the importance of both additive and non-additive genetic variance in the inheritance of these traits. The genotyped L3 and L2 were found to be the most promising per se for most of the trait, whereas the cross combination L3 x T3, L2 x T2 involving good x good general combiner parents was found with good desirable sca effects. The cross combination L7 x T1 was found with best SCA effect for T.S.S. To further improve yield and quality traits inclusion of F1 combinations with high SCA and parents with good GCA in multiple crosses, Line $x$ Tester mating could be a worthwhile approach.

\section{Introduction}

Cucumber is warm season vegetable grown throughout the world under tropical and subtropical conditions. It is said to be the native of northern India (Pursglove, 1969). The fruits of cucumber is said to have cooling effect, prevent constipation, checks jaundice and indigestion (Nandkarni, 1927).

Besides this, the seed of cucumber is also used in Ayurvedic preparations and raw fruits are being used for cosmetic purpose. Snap melon (Cucumis melo var. momordica) belongs to the family cucurbitaceae is used as a vegetable in variety of ways. Snap melon is rich in quality and now snapmelon juice is gaining popularity as squash. Knowledge of the nature and magnitude of variation promotes a rational choice of the characters in which selection can be exercised.

\section{Materials and Methods}

The present investigation entitled "Heterosis, Combining Ability and Stability in Interspecific Hybrids of Cucumis", was conducted during Kharif, 2014 at three different locations (Horticulture Farm, Department of Horticulture, Rajasthan College of Agriculture, Udaipur, Agricultural Research Station, Banswara and KVK Chittorgarh).

Twelve inbred lines (female) of cucumis melo were crossed with three testers of cucumis 
sativus in line $\mathrm{x}$ tester mating design to develop a total 36 hybrids at Hi-Tech Horticulture unit, Department of Horticulture, RajasthanCollege of Agriculture,Udaipur. These 15 parents along with 36 hybrids and three standard checks (Mamta-5002, Sedona, Kakri surya prabha) were evaluated in randomized block design with three replications at three locations viz. Udaipur, Banswara and Chittorhgarh during kharif 2014. Lines and testers accessions were collected from NBPGR, New Delhi (Table 1).

The observations were recorded for eighteen important characters namely vine length, number of branches per vine, days to anthesis of first female and male flower, number of male flower per vine, number of female flower per vine, sex ratio, number of fruits per vine, fruit weight fruit length, fruit volume, fruit diameter, pulp thickness, total yield per vine, pulp weight, seed weight (Table 2).

\section{Results and Discussion}

Analysis of variance for combining ability reflected significant difference among crosses for all the characters in all the environments, partitioning of this variance in lines, testers and line $\mathrm{x}$ testers revealed significant difference, among GCA of lines for all the characters, GCA of tester for all the characters except number of branches per vine in E1, specific gravity in E3.

Significant difference among SCA of hybrids was observed for all the characters in all the environments. In all the characters tester, lines and line $\mathrm{x}$ tester interacted with environments significantly except days to anthesis of first female flower, fruit weight and fruit length due to testers, this indicate lack of consistency in GCA of lines and tester and SCA of crosses across the environments. It suggested that selection of the parents and crosses for GCA and SCA in different environments should be done separately.

Analysis of GCA, SCA variance for fruit texture was reported by Yoshioka et al., (2010). Olfati et al., (2011), Bairagi et al., (2013) and reported that both GCA and SCA variance were important for yield, yield contributing characters, quality characters and for earliness.

The estimates of GCA effects revealed that the good general combiner for yield and yield contributing characters were lines L2, L3, L5, L8 and L12 for T.S.S. L7, L11, and L12 for fruit quality traits L2, L3, L5 and L12 for plant type trait L4, L9, L10 and L12 for flowering traits L1, L3, L6, L7, and L12 in general L2, L3, L5, L7 and L12 considered for good combiners for yield and majority of the traits (Table 3).

Among the tester the tester, T3 was considered good general combiner for yield, flowering and plant type traits.

The tester T2 was considered good general combiner for quality traits. The high general combining ability effects observed is due to additive gene effect and additive $\mathrm{x}$ additive gene effects (Griffing, 1956 and Sprague, 1966).

A perusal of SCA effects revealed that highest magnitude of positive significant SCA effects for total yield per vine were recorded in hybrids L2× T2 in E1 (3.10), in E3 (2.33) and on pooled basis (1.55), L10× T3 in E2 (2.06).

Two hybrids L10× T3 and L3× T3 exhibited positive significant SCA effects in all the three environments as well as on pooled basis for total yield per vine. 
Table.1 Description of parents

\begin{tabular}{llll}
\hline S.No & Symbol & Species Name & IC Number \\
\hline A. & Lines (Female Parents) & \\
1. & $\mathrm{~L}_{1}$ & C.melo var. momoradica & IC-415539 \\
2. & $\mathrm{~L}_{2}$ & C.melo var. momoradica & IC-415521 \\
3. & $\mathrm{~L}_{3}$ & C.melo var. momoradica & IC-433621 \\
4. & $\mathrm{~L}_{4}$ & C.melo var. utilissimus & IC-315294 \\
5. & $\mathrm{~L}_{5}$ & C.melo var. utilissimus & IC-258163 \\
6. & $\mathrm{~L}_{6}$ & C.melo var. utilissimus & IC-313031 \\
7. & $\mathrm{~L}_{7}$ & C.melo var. momoradica & VRSM-44 \\
8. & $\mathrm{~L}_{8}$ & C.melo var. agrertris & IC-258165 \\
9. & $\mathrm{~L}_{9}$ & C.melo var. momoradica & VRSM-32 \\
10. & $\mathrm{~L}_{10}$ & C.melo var. momoradica & DR/KPS/26 \\
11. & $\mathrm{~L}_{11}$ & C.melo & BS-41 \\
12. & $\mathrm{~L}_{12}$ & C.melo var. momoradica & VRSM-58 \\
B. & Tester (Male Parent) & \\
1. & $\mathrm{~T}_{1}$ & C.sativus & SKY/DR/RS \\
2. & $\mathrm{~T}_{2}$ & C.sativus & SPP-58 \\
3. & $\mathrm{~T}_{3}$ & C.sativus & SPP-56
\end{tabular}

Table.2 Grand Mean, Mean \pm SE (m) and range of eighteen characters in parents and F1

\begin{tabular}{lc|c|c|cc}
\hline \multirow{2}{*}{ Characters } & GM & \multicolumn{2}{c|}{ Parents } & \multicolumn{2}{c}{$\mathrm{F}_{1}$} \\
\cline { 2 - 6 } & & Mean $\pm \mathrm{SE}(\mathrm{m})$ & Range & Mean $\pm \mathrm{SE}(\mathrm{m})$ & Range \\
\hline Vine length & 2.39 & $2.68 \pm 0.09$ & $1.53-3.66$ & $2.31 \pm 0.09$ & $1.30-3.78$ \\
Number of branches & 4.31 & $4.01 \pm 0.20$ & $3.04-5.64$ & $4.41 \pm 0.20$ & $2.69-5.89$ \\
Days to anthesis of first male flower & 37.28 & $35.40 \pm 0.67$ & $31.80-39.09$ & $38.11 \pm 0.67$ & $34.31-42.47$ \\
Days to anthesis of first female flower & 43.49 & $41.63 \pm 0.81$ & $38.73-46.56$ & $44.37 \pm 0.81$ & $39.42-52.18$ \\
No. of male flower/vine & 132.90 & $137.56 \pm 2.27$ & $92.38-197.93$ & $131.31 \pm 2.27$ & $94.29-157.89$ \\
No. of female flower/vine & 16.52 & $16.32 \pm 0.70$ & $7.78-31.49$ & $16.54 \pm 0.70$ & $8.36-29.27$ \\
Sex ratio & 9.17 & $9.67 \pm 0.37$ & $4.86-14.44$ & $9.06 \pm 0.37$ & $5.10-16.72$ \\
Number of fruit/vine & 5.45 & $5.47 \pm 0.24$ & $3.18-11.98$ & $5.52 \pm 0.24$ & $2.96-11.98$ \\
Fruit weight & $611.30558 .99 \pm 26.63$ & $140.61-1441.06653 .74 \pm 26.63$ & $133.47-1385.67$ \\
Fruit diameter & 7.53 & $7.87 \pm 0.28$ & $3.28-14.89$ & $7.66 \pm 0.28$ & $4.40-11.82$ \\
Fruit length & 18.15 & $16.34 \pm 0.58$ & $6.94-24.26$ & $18.64 \pm 0.58$ & $7.91-34.30$ \\
Pulp thickness & 14.79 & $14.63 \pm 0.49$ & $5.79-23.84$ & $15.13 \pm 0.49$ & $8.00-24.28$ \\
T.S.S & 5.14 & $4.81 \pm 0.11$ & $3.73-6.18$ & $5.26 \pm 0.11$ & $3.76-8.17$ \\
Fruit volume & $736.03688 .04 \pm 20.40$ & $204.22-1675.33$ & $779.07 \pm 20.40$ & $146.22-1935.11$ \\
Specific gravity & 0.86 & $0.84 \pm 0.03$ & $0.72-1.03$ & $0.88 \pm 0.03$ & $0.71-1.03$ \\
Total yield/vine & 3.26 & $2.93 \pm 0.12$ & $0.46-6.98$ & $3.54 \pm 0.12$ & $1.04-7.73$ \\
Pulp weight & $491.06535 .25 \pm 16.62$ & $158.61-1656.37$ & $495.07 \pm 16.62$ & $120.65-1474.43$ \\
Seed weight & 120.16 & $126.18 \pm 4.03$ & $38.30-195.46$ & $123.71 \pm 4.03$ & $34.96-226.91$ \\
\hline
\end{tabular}


Table.3 GCA effects of parents

\begin{tabular}{ccccccccc}
\hline Parents & $\begin{array}{l}\text { Vine } \\
\text { length }\end{array}$ & $\begin{array}{l}\text { No. of } \\
\text { branches } \\
\text { per vine }\end{array}$ & $\begin{array}{l}\text { Days to } \\
\text { anthesis of } \\
\text { first male } \\
\text { flower }\end{array}$ & $\begin{array}{l}\text { Days to } \\
\text { anthesis of } \\
\text { first female } \\
\text { Flower }\end{array}$ & $\begin{array}{l}\text { No. of } \\
\text { male } \\
\text { lower per } \\
\text { vine }\end{array}$ & $\begin{array}{l}\text { No. of } \\
\text { female } \\
\text { flower per } \\
\text { vine }\end{array}$ & $\begin{array}{l}\text { Sex } \\
\text { ratio }\end{array}$ & $\begin{array}{l}\text { No. of } \\
\text { fruit per } \\
\text { vine }\end{array}$ \\
\hline T1 & $0.11^{* *}$ & -0.03 & $-1.03^{* *}$ & $-1.30^{* *}$ & $-2.33^{* *}$ & 0.42 & $-0.40^{* *}$ & $0.82^{* *}$ \\
T2 & $-0.41^{* *}$ & 0.00 & $0.68^{* *}$ & $1.10^{* *}$ & -1.19 & $-1.60^{* *}$ & $0.79^{* *}$ & $-0.43^{* *}$ \\
T3 & $0.30^{* *}$ & 0.03 & 0.35 & 0.20 & $3.53^{* *}$ & $1.18^{* *}$ & $-0.39^{* *}$ & $-0.39^{* *}$ \\
L1 & $-0.19^{* *}$ & -0.15 & $-0.99^{*}$ & $-2.20^{* *}$ & -0.68 & $-1.31^{* *}$ & 0.28 & -0.21 \\
L2 & -0.08 & $-0.97^{* *}$ & -0.50 & -0.46 & $4.37^{* *}$ & $-1.20^{* *}$ & $0.84^{* *}$ & 0.10 \\
L3 & $-0.15^{* *}$ & 0.11 & $-1.59^{* *}$ & $-1.68^{* *}$ & $14.63^{* *}$ & $2.79^{* *}$ & $-0.80^{* *}$ & $0.55^{* *}$ \\
L4 & $0.25^{* *}$ & $-0.58^{* *}$ & $0.80^{*}$ & -0.03 & $-26.68^{* *}$ & $-5.21^{* *}$ & $0.92^{* *}$ & $-1.37^{* *}$ \\
L5 & -0.07 & 0.14 & $1.30^{* *}$ & 0.23 & $8.55^{* *}$ & $2.23^{* *}$ & $-1.22^{* *}$ & $-0.73^{* *}$ \\
L6 & $-0.16^{* *}$ & 0.05 & $-1.84^{* *}$ & -0.71 & $-10.03^{* *}$ & $-4.14^{* *}$ & $1.40^{* *}$ & $-1.25^{* *}$ \\
L7 & $-0.26^{* *}$ & $0.33^{* *}$ & $-1.85^{* *}$ & -0.75 & $-11.11^{* *}$ & $3.63^{* *}$ & $-2.84^{* *}$ & $0.74^{* *}$ \\
L8 & $-0.87^{* *}$ & $-0.61^{* *}$ & $0.90^{*}$ & -0.80 & $-14.11^{* *}$ & $-3.79^{* *}$ & $1.38^{* *}$ & $-0.71^{* *}$ \\
L9 & $0.63^{* *}$ & $0.42^{* *}$ & $1.55^{* *}$ & $1.73^{* *}$ & $11.52^{* *}$ & $1.09^{* *}$ & $1.28^{* *}$ & -0.26 \\
L10 & $0.16^{* *}$ & $0.45^{* *}$ & $2.44^{* *}$ & $3.29^{* *}$ & $14.47^{* *}$ & $-1.33^{* *}$ & $1.69^{* *}$ & $-0.30^{* *}$ \\
L11 & 0.10 & $-0.45^{* *}$ & $1.62^{* *}$ & $1.90^{* *}$ & $15.99^{* *}$ & $3.08^{* *}$ & -0.28 & $3.35^{* *}$ \\
L12 & $0.65^{* *}$ & $1.26^{* *}$ & $-1.85^{* *}$ & -0.52 & $-6.91^{* *}$ & $4.16^{* *}$ & $-2.65^{* *}$ & 0.10 \\
\hline
\end{tabular}

\begin{tabular}{|c|c|c|c|c|c|c|c|c|c|c|}
\hline Parents & $\begin{array}{c}\text { Fruit } \\
\text { weight }\end{array}$ & $\begin{array}{c}\text { Fruit } \\
\text { diameter }\end{array}$ & $\begin{array}{l}\text { Fruit } \\
\text { length }\end{array}$ & $\begin{array}{c}\text { Pulp } \\
\text { thickness }\end{array}$ & T.S.S & $\begin{array}{c}\text { Fruit } \\
\text { volume }\end{array}$ & SG & $\begin{array}{l}\text { Total } \\
\text { yield/ } \\
\text { vine }\end{array}$ & $\begin{array}{c}\text { Pulp } \\
\text { weight }\end{array}$ & $\begin{array}{c}\text { Seed } \\
\text { weight }\end{array}$ \\
\hline $\mathrm{T} 1$ & $-22.79 *$ & -0.11 & $-2.81 * *$ & 0.05 & -0.01 & $-60.29 * *$ & $0.03 * *$ & $0.17 * *$ & $-46.64 * *$ & 0.35 \\
\hline $\mathrm{T} 2$ & $-48.87 * *$ & $-0.30 * *$ & 0.23 & $-0.55 * *$ & -0.00 & $-42.44 * *$ & $-0.02 *$ & $-0.28 * *$ & $-29.96 * *$ & $7.34 * *$ \\
\hline $\mathrm{T} 3$ & $71.66 * *$ & $0.41 * *$ & $2.58 * *$ & $0.50 * *$ & 0.01 & $102.73 * *$ & -0.01 & $0.11 * *$ & $76.60 * *$ & $-7.69 * *$ \\
\hline L1 & $-195.00 * *$ & $-1.49 * *$ & $-0.88^{*}$ & $-4.15 * *$ & $-0.27 * *$ & $-248.99 * *$ & 0.02 & $-1.03 * *$ & $-224.53 * *$ & -0.45 \\
\hline L2 & $226.97 * *$ & $1.21 * *$ & $2.86 * *$ & $0.93 * *$ & $-0.59 * *$ & $250.49 * *$ & 0.00 & $1.38 * *$ & $269.40 * *$ & $45.32 * *$ \\
\hline L3 & $443.45^{* *}$ & $2.51 * *$ & $4.45 * *$ & $5.19 * *$ & $-0.58 * *$ & $623.90 * *$ & -0.03 & $3.11 * *$ & $727.26^{* *}$ & $45.57 * *$ \\
\hline L4 & $-273.94 * *$ & $-2.61 * *$ & $5.13 * *$ & $-2.72 * *$ & -0.09 & $-272.85^{* *}$ & $-0.09 * *$ & $-2.06^{* *}$ & $-152.83 * *$ & $-42.98 * *$ \\
\hline L5 & $214.37 * *$ & $1.99 * *$ & -0.68 & $2.79 * *$ & -0.06 & $337.30 * *$ & $-0.08 * *$ & $0.68 * *$ & 12.71 & $16.97 * *$ \\
\hline L6 & 11.33 & $-1.87 * *$ & $8.78 * *$ & $-0.98 * *$ & $-0.28 * *$ & $104.49 * *$ & $-0.08 * *$ & $-0.67 * *$ & $-46.67 * *$ & $-20.46 * *$ \\
\hline L7 & $-213.40 * *$ & 0.25 & $-5.83 * *$ & $-1.26 * *$ & $0.90 * *$ & $-271.29 * *$ & $0.06^{* *}$ & $-0.81 * *$ & $-217.93 * *$ & $-24.77 * *$ \\
\hline L8 & $115.06^{* *}$ & $-0.49 * *$ & $0.79 *$ & 0.14 & 0.04 & $65.45^{* *}$ & $0.06 * *$ & $0.27 * *$ & $-101.52 * *$ & 8.66 ** \\
\hline L9 & 25.13 & $1.10^{* *}$ & $-2.51 * *$ & $2.48 * *$ & 0.07 & -7.14 & 0.02 & 0.02 & $-40.33 * *$ & $59.62 * *$ \\
\hline L10 & $-61.97 * *$ & -0.15 & $-2.24 * *$ & $0.88^{* *}$ & $-0.35 * *$ & $-97.22 * *$ & -0.01 & $-0.18^{*}$ & $-113.58^{* *}$ & $-20.08 * *$ \\
\hline L11 & $-502.37 * *$ & $-2.72 * *$ & $-9.99 * *$ & $-6.69 * *$ & $0.84 * *$ & $-607.59 * *$ & 0.02 & $-2.22 * *$ & $-355.06^{* *}$ & $-82.26 * *$ \\
\hline L12 & $210.37 * *$ & $2.27 * *$ & 0.12 & $3.39 * *$ & $0.38 * *$ & $123.45^{* *}$ & $0.11 * *$ & $1.50 * *$ & $243.08 * *$ & $14.87 * *$ \\
\hline
\end{tabular}


Table.4 SCA effects of hybrids

\begin{tabular}{|c|c|c|c|c|c|c|c|c|}
\hline Parents & $\begin{array}{l}\text { Vine } \\
\text { length }\end{array}$ & $\begin{array}{l}\text { No. of } \\
\text { branches } \\
\text { per vine }\end{array}$ & $\begin{array}{l}\text { Days to } \\
\text { anthesis } \\
\text { of first } \\
\text { male } \\
\text { flower }\end{array}$ & $\begin{array}{l}\text { Days to } \\
\text { anthesis } \\
\text { of first } \\
\text { female } \\
\text { flower }\end{array}$ & $\begin{array}{l}\text { No. of } \\
\text { male } \\
\text { flower } \\
\text { per vine }\end{array}$ & $\begin{array}{l}\text { No. of } \\
\text { female } \\
\text { flower } \\
\text { per vine }\end{array}$ & $\begin{array}{l}\text { Sex } \\
\text { ratio }\end{array}$ & $\begin{array}{l}\text { No. of } \\
\text { fruit per } \\
\text { vine }\end{array}$ \\
\hline L1 x T1 & $-0.58 * *$ & 0.19 & $2.04 *$ & 0.62 & $5.65^{*}$ & $3.57 * *$ & $-1.87 * *$ & -0.11 \\
\hline L2 $\times$ T1 & 0.05 & $-0.50^{*}$ & 0.62 & 0.66 & $6.79^{*}$ & 0.81 & -0.83 & -0.02 \\
\hline L3 x T1 & $-0.33 * *$ & $-1.01 * *$ & 1.20 & -0.04 & $14.28 * *$ & $-4.64 * *$ & $2.98 * *$ & $-1.07 * *$ \\
\hline L4 x T1 & 0.03 & 0.45 & $-2.02 *$ & -1.62 & -0.17 & $-2.55 * *$ & $1.99 * *$ & 0.37 \\
\hline L5 x T1 & -0.12 & -0.17 & -0.76 & $2.03 *$ & 1.59 & $-1.69 *$ & 0.83 & $-1.25 * *$ \\
\hline L6 x T1 & $0.24 *$ & -0.32 & -0.93 & -0.90 & $-24.66 * *$ & $-4.21 * *$ & $1.74 * *$ & -1.60 ** \\
\hline L7 x T1 & $0.22 *$ & 0.13 & 0.03 & 1.42 & -0.25 & $-3.46 * *$ & $1.32 * *$ & -0.10 \\
\hline L8 x T1 & -0.16 & $0.81 * *$ & -0.94 & -0.92 & -2.98 & $-3.58 * *$ & $2.16^{* *}$ & 0.50 \\
\hline L9 x T1 & $-0.47 * *$ & 0.35 & $2.21 * *$ & $1.96^{*}$ & 1.37 & -0.45 & $-1.45 * *$ & 0.30 \\
\hline L10 x T1 & $0.35 * *$ & 0.22 & 0.10 & 0.93 & -2.38 & $5.61 * *$ & $-3.48 * *$ & 0.48 \\
\hline L11 x T1 & $0.48^{* *}$ & -0.31 & -1.32 & -1.01 & 4.43 & $9.22 * *$ & $-3.16^{* *}$ & $2.29 * *$ \\
\hline L12 x T1 & $0.28 * *$ & 0.18 & -0.23 & $-3.12 * *$ & -3.67 & 1.34 & -0.23 & 0.21 \\
\hline L1 x T2 & 0.08 & $0.91 * *$ & $-2.33 * *$ & $-2.36 *$ & $-10.47 * *$ & 0.57 & -0.79 & $0.81 * *$ \\
\hline L2 2 T2 & 0.21 & $1.29 * *$ & $-1.71 *$ & $-2.82 * *$ & 0.85 & $4.70 * *$ & $-3.16^{* *}$ & $2.12 * *$ \\
\hline L3 x T2 & -0.03 & $0.72 * *$ & -0.53 & -0.35 & $-13.64 * *$ & $3.51 * *$ & $-2.49 * *$ & 1.21 ** \\
\hline L4 x T2 & $0.53 * *$ & $-0.52 *$ & -0.35 & 0.23 & $-5.89 *$ & $4.18 * *$ & $-3.33 * *$ & $-0.76 * *$ \\
\hline L5 x T2 & -0.02 & $-1.14 * *$ & $-1.73 *$ & $-4.28 * *$ & 1.49 & -0.40 & -0.17 & 0.56 \\
\hline L6 x T2 & $0.30 * *$ & 0.29 & 0.34 & 0.95 & $16.78^{* *}$ & $2.00 *$ & -0.35 & $1.12 * *$ \\
\hline L7 x T2 & -0.17 & -0.02 & 1.24 & -1.92 & $-6.88^{*}$ & $3.63 * *$ & $-1.91 * *$ & $0.66^{*}$ \\
\hline L8 x T2 & $0.28 *$ & $-1.07 * *$ & $2.27 * *$ & 0.07 & $13.73 * *$ & -0.33 & $0.90 *$ & $-1.09 * *$ \\
\hline L9 x T2 & $0.66 * *$ & $-0.60 *$ & 1.55 & $2.81 * *$ & $-6.28 *$ & $-7.67 * *$ & $5.59 * *$ & $-0.65^{*}$ \\
\hline $\mathrm{L} 10 \times \mathrm{T} 2$ & $-0.25^{*}$ & $-0.53 *$ & -0.52 & 0.69 & $10.21 * *$ & $-2.79 * *$ & $3.08 * *$ & $-0.87 * *$ \\
\hline $\mathrm{L} 11 \times \mathrm{T} 2$ & $-0.78 * *$ & $0.61 *$ & $2.06^{*}$ & $4.81 * *$ & -1.44 & $-4.75 * *$ & $2.18 * *$ & $-3.15 * *$ \\
\hline $\mathrm{L} 12 \times \mathrm{T} 2$ & $-0.81 * *$ & 0.06 & -0.29 & $2.17 *$ & 1.55 & $-2.66 * *$ & 0.44 & 0.04 \\
\hline L1 x T3 & $0.50 * *$ & $-1.10 * *$ & 0.29 & 1.74 & 4.81 & $-4.15 * *$ & $2.65 * *$ & $-0.70 *$ \\
\hline L2 x T3 & $-0.25^{*}$ & $-0.78 * *$ & 1.09 & $2.16^{*}$ & $-7.63 * *$ & $-5.51 * *$ & $3.99 * *$ & $-2.10 * *$ \\
\hline L3 x T3 & $0.36 * *$ & 0.29 & -0.66 & 0.39 & -0.63 & 1.13 & -0.49 & -0.14 \\
\hline L4 x T3 & $-0.57 * *$ & 0.08 & $2.36^{* * *}$ & 1.39 & $6.06^{*}$ & -1.63 & $1.33 * *$ & 0.39 \\
\hline L5 x T3 & 0.15 & $1.30 * *$ & $2.49 * *$ & $2.24 *$ & -3.08 & $2.08^{*}$ & -0.66 & $0.69 *$ \\
\hline L6 x T3 & $-0.54 * *$ & 0.04 & 0.59 & -0.06 & $7.88 * *$ & $2.21 * *$ & $-1.39 * *$ & 0.48 \\
\hline L7 x T3 & -0.05 & -0.11 & -1.27 & 0.49 & $7.13 * *$ & -0.18 & 0.59 & -0.56 \\
\hline L8 x T3 & -0.12 & 0.26 & -1.33 & 0.86 & $-10.75^{* *}$ & $3.91 * *$ & $-3.06 * *$ & $0.58 *$ \\
\hline L9 x T3 & -0.19 & 0.25 & $-3.76 * *$ & $-4.76 * *$ & 4.91 & $8.12 * *$ & $-4.13 * *$ & 0.35 \\
\hline L10 x T3 & -0.10 & 0.31 & 0.42 & -1.61 & $-7.82 * *$ & $-2.83 * *$ & 0.41 & 0.40 \\
\hline L11 x T3 & $0.30 * *$ & -0.30 & -0.74 & $-3.80 * *$ & -2.99 & $-4.47 * *$ & $0.97 *$ & $0.86^{* *}$ \\
\hline L12 x T3 & $0.52 * *$ & -0.24 & 0.53 & 0.96 & 2.12 & 1.31 & -0.21 & -0.25 \\
\hline
\end{tabular}




\begin{tabular}{|c|c|c|c|c|c|c|c|c|c|c|}
\hline Parents & \multirow{2}{*}{$\begin{array}{l}\begin{array}{l}\text { Fruit } \\
\text { weight }\end{array} \\
-28.27\end{array}$} & \multirow{2}{*}{$\begin{array}{l}\begin{array}{l}\text { Fruit } \\
\text { diame } \\
\text { ter }\end{array} \\
-0.61\end{array}$} & \multirow{2}{*}{$\begin{array}{r}\begin{array}{l}\text { Fruit } \\
\text { length }\end{array} \\
1.03\end{array}$} & \multirow{2}{*}{$\begin{array}{l}\begin{array}{l}\text { Pulp } \\
\text { thicknes } \\
\text { s }\end{array} \\
0.08\end{array}$} & \multirow{2}{*}{$\begin{array}{l}\text { T.S.S } \\
0.03\end{array}$} & \multirow{2}{*}{$\begin{array}{l}\begin{array}{l}\text { Fruit } \\
\text { volume }\end{array} \\
-43.12\end{array}$} & \multirow{2}{*}{$\begin{array}{l}\text { SG } \\
0.01\end{array}$} & \multirow{2}{*}{$\begin{array}{l}\begin{array}{l}\text { Total } \\
\text { yield/vin } \\
\text { e }\end{array} \\
-0.16\end{array}$} & \multirow{2}{*}{$\begin{array}{l}\begin{array}{l}\text { Pulp } \\
\text { weight }\end{array} \\
21.27\end{array}$} & \multirow{2}{*}{$\begin{array}{l}\begin{array}{l}\text { Seed } \\
\text { weight }\end{array} \\
-29.32 * *\end{array}$} \\
\hline L1 x T1 & & & & & & & & & & \\
\hline L2 2 T1 & $-92.10 * *$ & $-0.68 *$ & $1.78^{*}$ & $-5.51 * *$ & $0.27 *$ & $-116.15^{* *}$ & -0.01 & 0.03 & $-166.35^{* *}$ & $57.53 * *$ \\
\hline L3 x T1 & $\stackrel{-}{-}$ & $-1.07 * *$ & 1.10 & $3.92 * *$ & 0.03 & $-380.23^{* *}$ & $0.08 *$ & $-1.41 * *$ & $298.73 * *$ & $11.32 *$ \\
\hline L4 x T1 & -57.33 & 0.17 & -0.05 & -0.64 & -0.25 & $-107.93^{* *}$ & 0.05 & -0.19 & $-43.96^{*}$ & $-10.37^{*}$ \\
\hline L5 x T1 & $130.69 * *$ & $1.21 * *$ & $1.87 * *$ & $-1.78^{* *}$ & $-0.37 * *$ & $151.70 * *$ & 0.01 & -0.04 & $-176.85^{* *}$ & $-24.59 * *$ \\
\hline L6 x T1 & $146.30 * *$ & 0.12 & $-6.69 * *$ & $3.27 * *$ & 0.25 & $104.07 * *$ & 0.05 & -0.28 & $200.82 * *$ & -6.92 \\
\hline L7 x T1 & 3.52 & 0.03 & 0.22 & 0.67 & $2.02 * *$ & 9.40 & 0.03 & -0.10 & -4.95 & $-10.84 *$ \\
\hline L8 x T1 & 13.86 & -0.30 & 1.13 & -0.40 & -0.03 & 44.88 & -0.03 & $0.69 * *$ & -7.53 & 8.21 \\
\hline L9 x T1 & -10.21 & 0.24 & -1.33 & $1.51^{*}$ & $-0.33 *$ & -10.08 & 0.01 & 0.26 & $-67.02 * *$ & $-25.14 * *$ \\
\hline L10 x T1 & 36.94 & 0.37 & 0.43 & $-1.76^{* *}$ & $-0.36 * *$ & $181.77^{* *}$ & $-0.09 *$ & $0.59 * *$ & $-82.44 * *$ & 2.64 \\
\hline L11 x T1 & 4.89 & -0.43 & $2.83 * *$ & -0.49 & $-0.29 *$ & 35.03 & -0.01 & 0.12 & 27.27 & -6.84 \\
\hline $\mathrm{L} 12 \times \mathrm{T} 1$ & 3.06 & $0.96 * *$ & $-2.32 * *$ & 1.13 & $-0.97 * *$ & $130.66^{* *}$ & $0.10^{* *}$ & $0.51 * *$ & 1.02 & $34.32 * *$ \\
\hline $\mathrm{L} 1 \times \mathrm{T} 2$ & 11.12 & $0.73^{*}$ & $-3.01 * *$ & 0.56 & $0.82 * *$ & -39.19 & 0.05 & $0.29 *$ & 38.60 & $32.32 * *$ \\
\hline $\mathrm{L} 2 \times \mathrm{T} 2$ & -7.65 & -0.27 & 1.33 & 0.15 & $-0.90 * *$ & -16.90 & 0.03 & $1.55^{* *}$ & $-62.28 * *$ & $-32.64 * *$ \\
\hline $\mathrm{L} 3 \times \mathrm{T} 2$ & $-65.46^{*}$ & -0.17 & -1.01 & $-1.90 * *$ & 0.12 & $-49.19 *$ & -0.02 & $0.45^{* *}$ & $-164.85 * *$ & -3.62 \\
\hline $\mathrm{L} 4 \times \mathrm{T} 2$ & $131.12 * *$ & 0.33 & $3.38 * *$ & 0.75 & $-0.35^{* *}$ & $256.88^{* *}$ & $-0.09^{*}$ & 0.16 & $180.15^{* *}$ & $38.41 * *$ \\
\hline $\mathrm{L} 5 \times \mathrm{T} 2$ & $-88.01 * *$ & 0.09 & $-3.03 * *$ & $1.86^{* *}$ & -0.23 & $-144.60 * *$ & 0.02 & -0.22 & $137.84 * *$ & $17.08 * *$ \\
\hline L6 x T2 & 18.49 & 0.24 & $6.64 * *$ & $-1.33 *$ & -0.13 & $152.44 * *$ & -0.06 & $0.63 * *$ & $-61.55^{* *}$ & $19.79 * *$ \\
\hline $\mathrm{L} 7 \times \mathrm{T} 2$ & $84.79 * *$ & $0.68 *$ & $-2.70 * *$ & 0.89 & $-0.92 * *$ & $51.99 *$ & 0.01 & $0.61 * *$ & 31.99 & -1.15 \\
\hline L8 x T2 & $70.70 *$ & -0.03 & -1.08 & $1.63 * *$ & $0.26^{*}$ & 11.03 & 0.04 & $-0.72 * *$ & 16.53 & $-43.96 * *$ \\
\hline L9 x T2 & $97.03 * *$ & 0.34 & $2.99 * *$ & 0.38 & $0.33 *$ & $95.62 * *$ & 0.02 & -0.18 & $138.51 * *$ & $34.35^{* *}$ \\
\hline $\mathrm{L} 10 \times \mathrm{T} 2$ & $\begin{array}{c}- \\
286.90 * *\end{array}$ & $-1.16^{* *}$ & $-4.27 * *$ & $-3.17 * *$ & 0.20 & $-322.30 * *$ & -0.04 & $-2.04 * *$ & $-167.37 * *$ & $-18.64 * *$ \\
\hline $\mathrm{L} 11 \times \mathrm{T} 2$ & 51.30 & 0.28 & 0.50 & 0.34 & $0.26 *$ & $52.51 *$ & -0.01 & -0.23 & 21.67 & -6.71 \\
\hline $\mathrm{L} 12 \times \mathrm{T} 2$ & -16.54 & $-1.04 * *$ & 0.26 & -0.16 & $0.53 * *$ & -48.30 & 0.05 & $-0.31 *$ & $-109.23 * *$ & $-35.23 * *$ \\
\hline L1 x T3 & 17.15 & -0.11 & $1.97 * *$ & -0.64 & $-0.86 * *$ & $82.31 * *$ & -0.07 & -0.13 & $-59.87 * *$ & -3.00 \\
\hline $\mathrm{L} 2 \times \mathrm{T} 3$ & $99.74 * *$ & $0.96^{* *}$ & $-3.11 * *$ & $5.37 * *$ & $0.63 * *$ & $133.05^{* *}$ & -0.02 & $-1.58 * *$ & $228.63 * *$ & $-24.89 * *$ \\
\hline L3 x T3 & $216.83 * *$ & $1.24 * *$ & -0.09 & $-2.01 * *$ & -0.15 & $429.42 * *$ & -0.07 & $0.97 * *$ & $-133.88 * *$ & -7.69 \\
\hline $\mathrm{L} 4$ x T3 & $-73.80^{*}$ & -0.50 & $-3.33 * *$ & -0.11 & $0.60 * *$ & $-148.95^{* *}$ & 0.04 & 0.03 & $-136.18 * *$ & $-28.05^{* *}$ \\
\hline L5 x T3 & -42.68 & $-1.29 * *$ & 1.16 & -0.08 & $0.60 * *$ & -7.10 & -0.02 & 0.26 & 39.02 & 7.51 \\
\hline L6 x T3 & $\stackrel{-}{-}$ & -0.36 & 0.05 & $-1.95^{* *}$ & -0.12 & $-256.51 * *$ & 0.02 & $-0.35^{*}$ & $-139.27 * *$ & $-12.87 * *$ \\
\hline L7 x T3 & $-88.31 * *$ & $-0.71 *$ & $2.48 * *$ & $-1.56 * *$ & $-1.10 * *$ & $-61.40 *$ & -0.04 & $-0.51 * *$ & -27.04 & $11.99 *$ \\
\hline L8 x T3 & $-84.56 * *$ & 0.33 & -0.05 & $-1.23^{*}$ & -0.23 & $-55.91^{*}$ & -0.02 & 0.03 & -9.00 & $35.75 * *$ \\
\hline L9 x T3 & $-86.82 * *$ & -0.57 & $-1.66^{*}$ & $-1.89 * *$ & -0.01 & $-85.54 * *$ & -0.03 & -0.08 & $-71.49 * *$ & -9.21 \\
\hline L10 x T3 & $249.95 * *$ & $0.78 *$ & $3.84 * *$ & $4.92 * *$ & 0.16 & $140.53 * *$ & $0.13 * *$ & $1.46^{* *}$ & $249.81 * *$ & $16.00 * *$ \\
\hline L11 x T3 & -56.19 & 0.14 & $-3.33 * *$ & 0.14 & 0.03 & $-87.54 * *$ & 0.02 & 0.10 & $-48.93 *$ & $13.55 * *$ \\
\hline L12 x T3 & 13.48 & 0.09 & $2.06 * *$ & -0.97 & 0.44 & $1 * * \quad-82.36 * *$ & & .05 & $.20 \quad 108.2$ & $21^{* *}$ \\
\hline
\end{tabular}


Hybrid L2× T2 besides total yield per vine also exhibited significant positive SCA effects for number of fruits per vine, number of female flower, and number of branches per vine. Hybrid L10× T3 in addition to yield per vine also exhibited significant positive SCA effects for fruit weight, fruit diameter, fruit length, pulp thickness, fruit volume, specific gravity, and pulp weight. Hybrid L3× T3 besides yield per vine also exhibited significant positive SCA effects for fruit volume, fruit weight, fruit diameter and for vine length. Hybrid L12× T1 in addition to yield per vine also exhibited significant positive SCA effects for vine length, fruit diameter and fruit volume (Table 4).

Out of total thirty six hybrids five best hybrids which exhibited highest positive significant SCA effect on pooled basis for total yield per vine were viz., L2x T2, L10x T3, L3x T3, L8x T1 and L6x T2. These hybrids also exhibited higher magnitude of economic heterosis with high mean performance. Similar findings for identification of superior parental lines, tester and hybrids based on GCA and SCA effects for fruit yield and morphological characters in cucumber were reported by Golabadi et al., (2015), Tasdighi and Baker (1981), Musmade et al., (1986) and Kumar et al., (2013) for yield and its components and for fruit texture by Yoshioka et al., (2010).

Out of total thirty six hybrids five best hybrids exhibited highest magnitude of positive significant SCA effects for T.S.S. on pooled basis were viz., L7× T1, L1× T2, L2× T3, L4× T3, and L5× T3 (Table 4). Similarly five best hybrids exhibiting highest positive significant SCA effects for fruit weight are viz., L10× T3, L3× T3, L6× T1, L4× T2, and L5 $\times$ T1.

On the basis of SCA/GCA effect, per se performance, economic heterosis, heterosis, heterobeltiosis best three economic hybrids were identified i.e. L3 x T3, L7 xT1, L4 x T2 for total yield per vine, T.S.S and fruit length respectively.

The mean performance of crosses could be envisaged as a criterion of SCA effect and selection of promising crosses based on per se performance. The present finding corroborated the earlier work of (Musmade et al., 1986, Srivastava and Srivastava, 1976; and Tasdighi and Baker, 1981). From the results of this experiment, it may be suggested that it is possible to predict the best hybrid combination for yield from the GCA values of the parental lines involved; at least in this population. Therefore, it may be concluded that hybrid breeding programme aimed at yield improvement in cucumber should be based on high GCA for yield in parental arrays (Bairagi et al., 2013).

\section{References}

Bairagi, S.K., Ram, H.H. and Singh, D.K. 2013. Analysis of combining ability in cucumber (cucumis sativus L.) through half diallel mating system. Annals of Horticulture, 6(2): 308-314.

Golabadi, M., Golkar, P. and Eghtedary, A. 2015. Combining ability analysis of fruit yield and morphological traits in greenhouse cucumber (Cucumis sativus L.). Canadian J. Plant Sci., 95(2): 377385.

Griffing, B. 1956. Concept of general and specific combining ability in relation to diallel crossing system. Australian J. Biol. Sci., 9: 463-493.

Kumar, J., Munshi, A.D., Kumar, R., Sureja, A.K. and Sharma, R.K. 2013. Combining ability and its relationship with gene action in slicing cucumber. Indian J. Horticulture, 70(1): 135-138.

Musmade, A.M. and Kale, P.N. 1986. Heterosis and combining ability in 
cucumber (Cucumis sativus L.). Veg. Sci., 13(1): 60-68.

Nandkarni, K.M. 1927. Indian materia medica. Nandkarni and Co. Bombay.

Olfati, J.A., Samizadeh, H. and Rabiei, B. 2011. Griffing's methods comparison for general and specific combining ability in cucumber. J. Biometrical Biostat., 2: 4.

Pursglove, J.W. 1969. Tropical crops dicotyledons I. longamans. Green and co. Ltd. London and Harlow.

Sprague, G.F. 1966. Quantitative genetics in plant improvement in plant breeding. A. symposium held at the Lowa state university (K.J. Fery, Ed.): 315-354.
Srivastava, V.K. and Srivastava, L.S. 1976. Genetic parameters, correlation coefficient and path coefficient analysis in bittergourd. Indian J. Horticulture, 33: 66-70.

Tasdighi, M. and Baker, L.R. 1981. Combining ability for femaleness and yield in single and 3-way crosses of pickling cucumber intended for onceover harvest. Euphytica, 30: 183-192.

Yoshioka, Y., Sugiyama, M. and Sakata, Y. 2010. Combining ability analysis of fruit texture traits in cucumber by mechanical measurement. Breeding Sci., 60: 65-70.

\section{How to cite this article:}

Sheetal Tak, R.A. Kaushik, K.D.Ameta, R.B. Dubey, R.S.Rathore, Anamika Nath. 2017. Combining ability studies on Cucumber and Snapmelon hybrids. Int.J.Curr.Microbiol.App.Sci. 6(6): 942-949. doi: https://doi.org/10.20546/ijcmas.2017.606.110 